\title{
The global effect of maternal education on complete childhood vaccination: a systematic review and meta-analysis
}

\author{
Jennifer Forshaw' ${ }^{1}$ Sarah M. Gerver ${ }^{1}$, Moneet Gill', Emily Cooper², Logan Manikam ${ }^{3^{*}}$ (D) and Helen Ward
}

\begin{abstract}
Background: There is an established correlation between maternal education and reduction in childhood mortality. One proposed link is that an increase in maternal education will lead to an increase in health care access and vaccine uptake. Vaccinations are a central preventative child health tool, therefore demonstrating the importance of understanding factors that can improve coverage. This review aims to establish if there is a correlation between increasing maternal education and vaccine uptake and if this varies between continents, setting and time.

Methods: An electronic database search was conducted using Medline Ovid, Embase and The Cochrane Library using a combination of keywords and appropriate MeSH terms for maternal education and child vaccination. Bibliographies were also hand searched. Data was extracted and entered onto a Microsoft Excel spreadsheet and analysed using STATA 13.0 software. The primary outcome of effect size of maternal education on completion of childhood vaccinations was analysed at different levels. Secondary outcomes were explored using subgroup analyses of differences between continents, rural or urban settings, and dates.
\end{abstract}

Results: The online search yielded 3430 papers, 37 were included in this study. The analysis showed increasing child vaccination uptake with increasing maternal education. Overall, analysis showed that the odds of full childhood vaccination were 2.3 times greater in children whose mother received secondary or higher education when compared to children whose mother had no education. There was large variability in the effect size between the studies included.

Conclusions: Improving maternal education is important for increasing childhood vaccination uptake and coverage. Further research is needed in higher income countries.

Trial registration: PROSPERO Registration No: CRD42016042409.

Keywords: Maternal education, Child health, Vaccination, Immunisation

\section{Background}

Despite the fact more children than ever are being vaccinated, millions of children each year fail to receive the complete routine immunization schedule [1]. Although the reason for this is likely multifactorial, it has been demonstrated that there is an association between maternal education and vaccination uptake $[2,3]$.

Childhood vaccinations are imperative for decreasing childhood mortality [1]. For this reason, global initiatives such as the Expanded Program on Immunization (EPI)

\footnotetext{
* Correspondence: Logan.manikam.10@ucl.ac.uk

${ }^{3} \mathrm{UCL}$ Great Ormond Street Institute of Child Health, 30 Guildford Street,

London WC1N 1EH, UK

Full list of author information is available at the end of the article
}

and the Global Alliance for Vaccine and Immunization (GAVI) have been put in place, outlining essential vaccinations and reinforcing their uptake [4-6]. Despite this, it is estimated that 1.5 million children under 5 years die from vaccine-preventable diseases annually [7]. Although literature has shown low caregiver education to be a common variable for under or non-immunization of children, there is no research to confirm whether it is a consistent finding and the overall effect size has not been established $[2,3,8]$.

The main aim of this study was to establish the global effect of maternal education on childhood vaccination in those under 12 years by quantifying the association between increasing maternal education and vaccine coverage in 
children, and assessing the variation in effect of maternal education by continent, setting, and over time.

\section{Methods \\ Protocol, eligibility criteria, information sources and search}

Medline, Embase, and the Cochrane Library were electronically searched on the 29th June 2016 using a combination of keywords and MeSH terms describing maternal education and child vaccination uptake. The search was restricted to English language and limited to those published between 1990 and 2016.

\section{Study selection, data collection and data items}

Observational studies of mothers with children under 12 years were included. Studies had an exposure variable of maternal education which is cross comparable such as "level of schooling achieved" or "literate versus illiterate" with a comparison group within the article.

The primary outcome assessed was completion of the full national or EPI schedule. Secondary outcomes were difference between continents, settings and dates.

Studies were subject to the following exclusion criteria: vaccine uptake not presented as raw, unadjusted data; unable to access the full text; review or narrative design; random control trials; case control trials not proportionate to the total population; studies where the exposure was another variable but maternal education was adjusted for in the analysis; studies with the outcome of specific vaccines, receipt of any vaccine, or vaccines not in the EPI.

Two authors (JF and MG, or EC and MG) independently screened all the titles. Abstracts were reviewed of potentially relevant articles, and full texts were retrieved to ascertain whether the inclusion criteria were fully met. Discrepancies were discussed until a consensus was reached. Data was extracted from included papers regarding study characteristics, including publication information (author and year), study country, setting, design, period, population total, children's age, maternal education parameter and vaccine types. The number of children per maternal education level, the number of children fully vaccinated per maternal education level, and the percentage of children fully vaccinated per maternal education level were extracted for data analysis.

When the paper presented more than one set of results, for example different years, locations or age-groups, the paper was split into alphabetically ordered groups. For the 2 cohort studies included, the oldest age followed in the study was used (7 months old).

\section{Risk of bias}

Papers were assessed for quality and risk of bias using an adapted version of the certified "Quality Assessment Tool for Quantitative Studies" by the Effective Public Health Practice Project (EPHPP) [9-11]. Each study was assessed according to the representativeness of the sample, study design, controlling of confounders, blinding of exposure for cohort studies, data collection measurements, and reporting of withdrawals and drop outs for cohort studies. The articles were given a global rating of strong, moderate or weak. All studies were kept in regardless of quality due to the small number of studies available and recognition of the limitations of the scoring systems $[10,12]$.

\section{Summary measures and synthesis of results}

For the meta-analysis the maternal education variables were collapsed into a binary categorical variable ("none/primary" and "secondary/higher"). In papers where there were only two categories for maternal education level and the level of education and the type of schooling received was not clear, i.e. "illiterate versus literate", "not educated versus educated", the educated variable was classified as "none/primary" as the level of education was not stated. For the six studies that divided papers into the categories "literate" and "illiterate" a separate meta-analysis was conducted for comparison. This is because the quality of education within countries can be highly varied, meaning we cannot conclude that a primary level education will result in maternal literacy [13]. Papers were excluded from the meta-analysis if the lowest level of education category included were "primary / secondary," "<high school," or "<12 years."

A pooled odds ratio, using the collapsed categories from each included paper, was calculated using a DerSimonianLaird [14] random effects model, as large heterogeneity was anticipated considering the differences in study characteristics, such as varied populations, healthcare, settings and education systems. The analysis was performed in Stata version 13.0 [15].

Sub-group analysis was also conducted for continent, setting, and for date the study was conducted. For the setting sub-group analysis, studies which were performed at a national or regional level were removed. In the date sub-group analysis, the data set was divided into two groups based upon the year that the studies were conducted, before and after 2000 to coincide with the release of the Millennium Development Goals.

All of the extracted papers were included into the pooled estimate analysis. The maternal education levels quoted in the papers were categorised into none, primary, secondary or tertiary to get an overall percentage of children fully vaccinated for each level.

Where dichotomous variables were stated, the lowest level was taken as this was the minimum amount the woman had received. Variables of "can read and write", "literate" and "mother educated" were categorised as primary as these skills can be achieved from primary school level. Where the paper included a variable with "less than", the 
country setting was taken into consideration due to variations in levels of mandatory education between countries.

Forest plots were created for the overall analyses and for each of the stratified analyses. These showed the individual study odds ratios and 95\% Confidence Intervals, the DerSimmonian-Laired pooled estimate and the $\mathrm{I}^{2}$-value for heterogeneity.

\section{Publication bias}

A scatter plot of number of children included in the studies against the prevalence of fully vaccinated children was created using STATA to assess for publication bias of the included papers.

\section{Results}

\section{Study selection}

The online search yielded 3430 results. Titles and abstracts were screened and duplicates or irrelevant articles were removed. In total, 218 full texts were retrieved and screened, with 37 articles being included in this review. Reasons for exclusion are outlined in Fig. 1, with the main reason being a lack of raw data.

Four papers were excluded from the meta-analysis as the lowest level of education was higher than primary.

\section{Study range and characteristics}

Of the 37 included papers, 35 were cross-sectional studies, the remaining 2 were cohort studies. All of the data from the studies was conducted between 1989 and 2013. India had eight studies, which is the greatest total number of studies per country. When assessing by continent, 18 were undertaken in Africa, 12 in Asia, three in Europe, three in North America and one in South America. This showed a dominance of research in lower income countries. The majority of the studies were regional or national, but six studies were set in urban areas, five in rural and one study

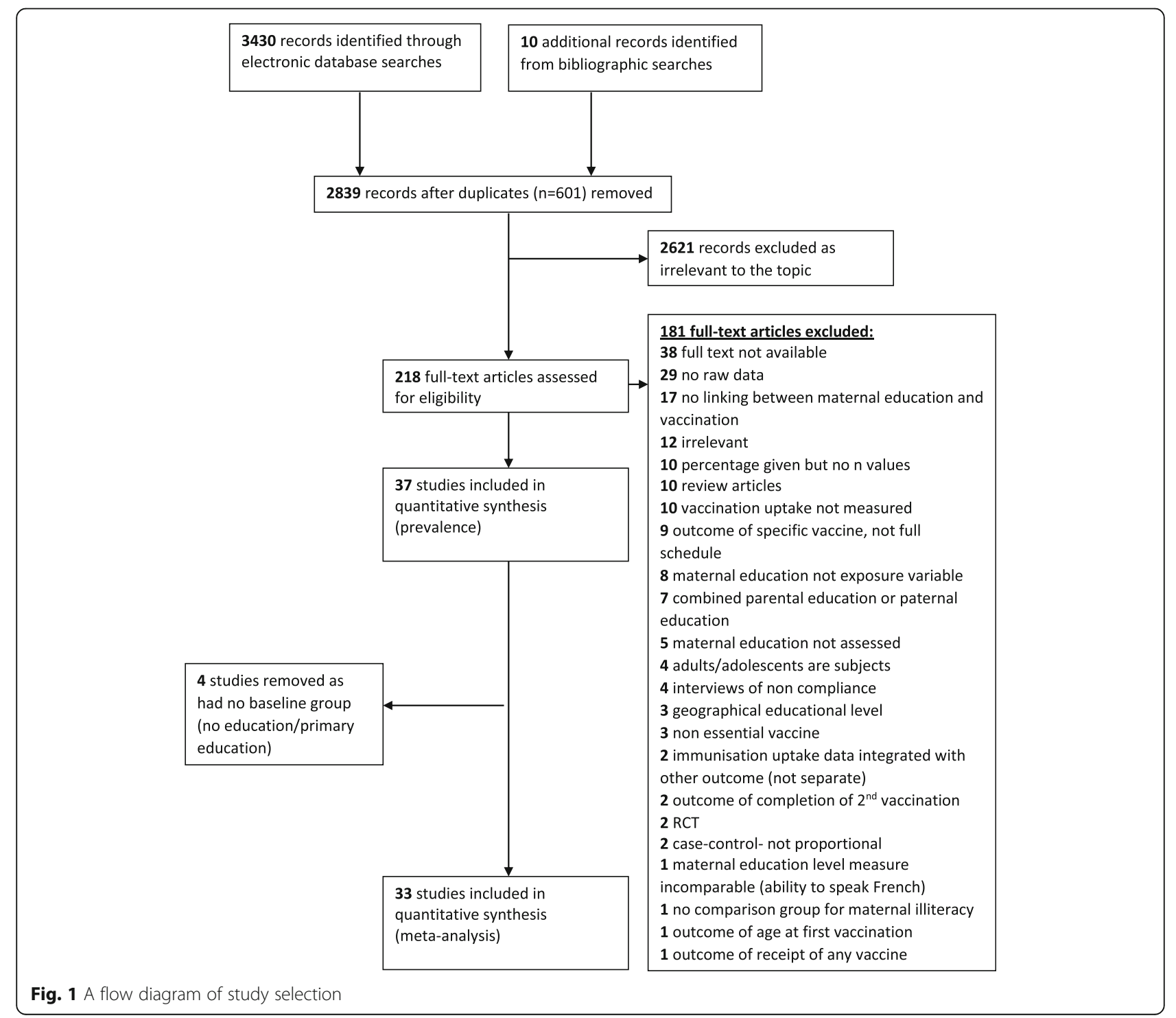


Table 1 Study characteristics

\begin{tabular}{|c|c|c|c|c|c|c|c|c|c|}
\hline Reference & Country & $\begin{array}{l}\text { Study } \\
\text { setting }\end{array}$ & $\begin{array}{l}\text { Study } \\
\text { design }\end{array}$ & $\begin{array}{l}\text { Study } \\
\text { period }\end{array}$ & Population & $\begin{array}{l}\text { Children's } \\
\text { age }\end{array}$ & Vaccine type & $\begin{array}{l}\text { Maternal } \\
\text { education } \\
\text { parameter }\end{array}$ & Quality \\
\hline $\begin{array}{l}\text { Al-Sheikh et al. } \\
\text { 1999a [17] }\end{array}$ & Iraq & Urban & Cross-sectional & 1989-1994 & $\begin{array}{l}341 \text { families } \\
\text { (186 urban), } \\
662 \text { children } \\
\text { (326 urban) }\end{array}$ & $0-2$ years & $\begin{array}{l}\text { BCG, DPT-OPV(3), } \\
\text { measles, MMR, } \\
\text { DPT-OPV(1 st booster) }\end{array}$ & $\begin{array}{l}\text { Illiterate; Reads } \\
\text { and writes; } \\
\text { Primary; } \\
\text { Intermediate; } \\
\text { Secondary; } \\
\text { Institute; College; } \\
\text { Postgraduate }\end{array}$ & Weak \\
\hline $\begin{array}{l}\text { Al-Sheikh et al. } \\
\text { 1999b [17] }\end{array}$ & Iraq & Rural & Cross-sectional & 1989-1994 & $\begin{array}{l}341 \text { families } \\
\text { (155 rural), } \\
662 \text { children } \\
\text { ( } 336 \text { rural) }\end{array}$ & $0-2$ years & $\begin{array}{l}\text { Completion of BCG, } \\
\text { DPT-OPV(3), measles, } \\
\text { MMR, DPT-OPV } \\
\text { (1st booster) }\end{array}$ & $\begin{array}{l}\text { Illiterate; Reads } \\
\text { and writes; } \\
\text { Primary; } \\
\text { Intermediate; } \\
\text { Secondary; } \\
\text { Institute; College; } \\
\text { Postgraduate }\end{array}$ & \\
\hline $\begin{array}{l}\text { Animaw et al., } \\
2014 \text { [24] }\end{array}$ & Ethiopia & Region & Cross-sectional & March 2013 & 630 children & $12-23$ months & $\begin{array}{l}1 \text { dose } B C G, 3 \text { doses } \\
\text { Polio, } 3 \text { doses } \\
\text { Pentavalant, } 3 \text { doses } \\
\text { PCV, } 1 \text { dose Measles }\end{array}$ & $\begin{array}{l}\text { None; Primary } \\
\text { school; High } \\
\text { school }\end{array}$ & Moderate \\
\hline Antai 2009 [4] & Nigeria & National & Cross-sectional & 2003 & $\begin{array}{l}\text { Interviews } \\
\text { from } 3725 \\
\text { women aged } \\
15 \text { to } 49 \text { years } \\
\text { with } 6029 \text { live } \\
\text { born children }\end{array}$ & $\begin{array}{l}12 \text { months } \\
\text { and older }\end{array}$ & $\begin{array}{l}\text { BCG, Polio (3), } \\
\text { DPT (3) } \\
\text { and Measles } \\
\text { vaccinations }\end{array}$ & $\begin{array}{l}\text { No education; } \\
\text { Primary; } \\
\text { Secondary } \\
\text { or higher }\end{array}$ & Moderate \\
\hline Antai 2012 [20] & Nigeria & National & Cross-sectional & 2008 & $\begin{array}{l}24,910 \\
\text { women aged } \\
15-49 \text { years } \\
\text { with live-born } \\
\text { children within } \\
5 \text { years before } \\
\text { the survey }\end{array}$ & $\begin{array}{l}12 \text { months } \\
\text { to } 5 \text { years }\end{array}$ & $\begin{array}{l}8 \text { childhood } \\
\text { vaccinations in the } \\
\text { EPI - BCG, DPT } 3 \\
\text { doses, OPV } 3 \text { doses, } \\
\text { and measles vaccine }\end{array}$ & $\begin{array}{l}\text { No education; } \\
\text { Primary school; } \\
\text { Secondary } \\
\text { school or higher }\end{array}$ & Moderate \\
\hline $\begin{array}{l}\text { Bbaale et al. } \\
2013 \text { [25] }\end{array}$ & Uganda & National & Cross-sectional & 2006 & 7591 children & 12-36 months & $\begin{array}{l}\text { Full vaccination, } \\
\text { BCG, DPT, Polio, } \\
\text { Measles vaccinations }\end{array}$ & $\begin{array}{l}\text { None, primary, } \\
\text { secondary, } \\
\text { post-secondary }\end{array}$ & Moderate \\
\hline $\begin{array}{l}\text { Branco et al. } \\
2014 \text { [26] }\end{array}$ & Brazil & Urban & Cross-sectional & January 2010 & 282 children & $12-59$ months & $\begin{array}{l}1 \text { dose BCG, } 3 \text { doses } \\
\text { Hep B, } 3 \text { doses } \\
\text { DTP-Hib, } 3 \text { doses } \\
\text { OPV, } 2 \text { doses } \\
\text { Rotavirus, } 1 \text { dose } \\
\text { Yellow fever, } \\
1 \text { dose MMR }\end{array}$ & $\begin{array}{l}0-8 \text { years of } \\
\text { schooling; > } \\
8 \text { years of } \\
\text { schooling }\end{array}$ & Moderate \\
\hline $\begin{array}{l}\text { Brenner et al. } \\
2001[27]\end{array}$ & USA & Urban & Cohort & $\begin{array}{l}\text { August } \\
1995 \text { to } \\
\text { September } \\
1996\end{array}$ & $\begin{array}{l}369 \text { singleton } \\
\text { births from } 3 \\
\text { hospitals from } \\
\text { low-income, } \\
\text { inner-city } \\
\text { patients }\end{array}$ & $\begin{array}{l}\text { Cohort followed } \\
\text { until } 7 \text { months }\end{array}$ & $\begin{array}{l}\text { UTD at } 7 \text { months if } \\
\text { had received } 3 \text { DTP, } \\
3 \text { HIB, and } 2 \text { polio } \\
\text { vaccinations }\end{array}$ & $\begin{array}{l}<12 \text { years; } \\
\geq 12 \text { years }\end{array}$ & Strong \\
\hline $\begin{array}{l}\text { Calhoun et al. } \\
2014 \text { [28] }\end{array}$ & Kenya & Region & Cross-sectional & $\begin{array}{l}\text { June-July } \\
2003\end{array}$ & 244 children & 12-23 months & $\begin{array}{l}3 \text { doses Polio, } 1 \text { dose } \\
\text { BCG, } 1 \text { dose Measles, } \\
3 \text { doses DPT or } \\
\text { pentavalent }\end{array}$ & $\begin{array}{l}\text { Years of } \\
\text { schooling: } 0-8,8 \\
\text { or more }\end{array}$ & Moderate \\
\hline $\begin{array}{l}\text { Chhabra et al. } \\
2007 \text { [29] }\end{array}$ & India & Urban & Cross-sectional & $\begin{array}{l}\text { October } \\
2003 \text { to } \\
\text { January } \\
2004\end{array}$ & 693 children & 24-47 months & $\begin{array}{l}\text { BCG, DPT and OPV } \\
\text { ( } 3 \text { primary and } \\
\text { booster), measles } \\
\text { and MMR }\end{array}$ & $\begin{array}{l}\text { Nil; } ;-8 \text { years; } \\
>8 \text { years }\end{array}$ & Moderate \\
\hline $\begin{array}{l}\text { Danis et al. } \\
2010 \text { [18] }\end{array}$ & Greece & National & Cross-sectional & $\begin{array}{l}\text { Academic year } \\
\text { 2004-2005 }\end{array}$ & $\begin{array}{l}3609 \text { parent/ } \\
\text { guardian-child pairs } \\
3434 \text { pairs in } \\
\text { the final analysis. } \\
\text { Children in first } \\
\text { year of Greek } \\
\text { grammar school }\end{array}$ & $\begin{array}{l}6-7 \text { years } \\
\text { (Mean age } \\
6.76 \text { years) }\end{array}$ & $\begin{array}{l}5 \text { doses of DTP vaccine, } 5 \\
\text { doses of poliomyelitis } \\
\text { vaccine, } 2 \text { doses of MMR } \\
\text { vaccine, } 3 \text { doses of HBV } \\
\text { vaccine and full } \\
\text { vaccination for Hib }\end{array}$ & $\begin{array}{l}<9 \text { years; } \\
9-11 \text { years; } \\
12 \text { years (high } \\
\text { school); } \\
\text { College/ } \\
\text { university graduate }\end{array}$ & Moderate \\
\hline $\begin{array}{l}\text { Elliott et al. } \\
\text { 2006a [30] }\end{array}$ & India & Rural & Cross-sectional & $\begin{array}{l}\text { September } \\
2003\end{array}$ & 470 families & 9 months & $\begin{array}{l}\text { BCG, OPV (4), } \\
\text { DPT (3) and measles }\end{array}$ & Illiterate; Literate & Weak \\
\hline $\begin{array}{l}\text { Elliott et al. } \\
2006 \mathrm{~b} \text { [30] }\end{array}$ & India & Rural & Cross-sectional & $\begin{array}{l}\text { September } \\
2003\end{array}$ & 470 families & 18 months & $\begin{array}{l}\text { BCG, OPV (5), } \\
\text { DPT (4) and measles }\end{array}$ & Illiterate; Literate & \\
\hline $\begin{array}{l}\text { Elliott et al. } \\
\text { 2006c [30] }\end{array}$ & India & Rural & Cross-sectional & $\begin{array}{l}\text { September } \\
2003\end{array}$ & 470 families & 6 years & $\begin{array}{l}\text { BCG, OPV (5), } \\
\text { DPT (4), measles and DT }\end{array}$ & Illiterate; Literate & \\
\hline $\begin{array}{l}\text { Fatiregun et al. } \\
2012 \text { [31] }\end{array}$ & Nigeria & Region & Cross-sectional & 2007 & $\begin{array}{l}540 \text { interviews, } 525 \\
\text { respondents }\end{array}$ & $12-23$ months & & & Moderate \\
\hline
\end{tabular}


Table 1 Study characteristics (Continued)

\begin{tabular}{|c|c|c|c|c|c|c|c|c|c|}
\hline Reference & Country & $\begin{array}{l}\text { Study } \\
\text { setting }\end{array}$ & $\begin{array}{l}\text { Study } \\
\text { design }\end{array}$ & $\begin{array}{l}\text { Study } \\
\text { period }\end{array}$ & Population & $\begin{array}{l}\text { Children's } \\
\text { age }\end{array}$ & Vaccine type & $\begin{array}{l}\text { Maternal } \\
\text { education } \\
\text { parameter }\end{array}$ & Quality \\
\hline & & & & & $\begin{array}{l}\text { mothers of } \\
\text { children }\end{array}$ & & $\begin{array}{l}\text { BCG, dose of measles, three } \\
\text { doses }(1,2,3) \text { of DPT, four doses } \\
(0-3) \text { of OPV }\end{array}$ & $\begin{array}{l}\text { Primary/ } \\
\text { secondary; } \\
\text { Post secondary }\end{array}$ & \\
\hline $\begin{array}{l}\text { Fatiregun et al. } \\
2013 \text { [32] }\end{array}$ & Nigeria & Region & Cross-sectional & 2006 & 1178 mothers & 12-23 months & $\begin{array}{l}\text { BCG, } 4 \text { doses OPV, } 3 \text { doses } \\
\text { DPT, } 3 \text { doses Hetaptitis B }\end{array}$ & $\begin{array}{l}\text { Tertiary education; } \\
\text { Secondary } \\
\text { education; Primary } \\
\text { education; None }\end{array}$ & Moderate \\
\hline $\begin{array}{l}\text { Huq et al. } \\
2008 \text { [33] }\end{array}$ & Bangladesh & National & Cross-sectional & 1999-2000 & 755 children & 12-23 months & $\begin{array}{l}\text { BCG and measles vaccinations } \\
\text { and all } 3 \text { doses of the DPT } \\
\text { and polio vaccines }\end{array}$ & $\begin{array}{l}\text { Below primary; } \\
\text { Secondary; } \\
\text { Higher secondary }\end{array}$ & Moderate \\
\hline $\begin{array}{l}\text { Jahn et al. } \\
2008 \text { [34] }\end{array}$ & Malawi & Rural & Cross-sectional & $\begin{array}{l}\text { 21st August } \\
2002 \text { to } 22 \text { nd } \\
\text { July } 2004\end{array}$ & 5418 children & $\begin{array}{l}\text { Under } 5 \text { years } \\
\text { old }\end{array}$ & $\begin{array}{l}\text { BCG, OPV3, DPT3 and measles } \\
\text { vaccine before their } \\
\text { 1st birthday }\end{array}$ & $\begin{array}{l}<5 \text { years primary; } \\
\text { Primary } 5 \text { + years; } \\
\text { Sec./tert. }\end{array}$ & Moderate \\
\hline $\begin{array}{l}\text { Kidane et al. } \\
2003[35]\end{array}$ & Ethiopia & Region & Cross-sectional & 2000 & 220 households & $12-23$ months & $\begin{array}{l}\text { BCG, measles, } \\
3 \text { doses of DPT/OPV }\end{array}$ & Illiterate; Literate & Weak \\
\hline $\begin{array}{l}\text { Koumaré et al. } \\
2009[36]\end{array}$ & Mali & Region & Cross-sectional & July 2006 & 750 children & 12-23 months & $\begin{array}{l}\text { BCG, DTCP1, DTCP2, } \\
\text { and DTCP3 and measles }\end{array}$ & $\begin{array}{l}\text { Mother not } \\
\text { educated; } \\
\text { Mother educated }\end{array}$ & Weak \\
\hline $\begin{array}{l}\text { Kumar et al. } \\
2010[37]\end{array}$ & India & $\begin{array}{l}\text { Hospital/ } \\
\text { Urban }\end{array}$ & Cross-sectional & $\begin{array}{l}\text { April to July } \\
2007\end{array}$ & $\begin{array}{l}325 \text { children ( } 148 \\
\text { males, } 177 \text { females) } \\
\text { admitted to } \\
\text { paediatrics ward at } \\
\text { a tertiary care } \\
\text { hospital }\end{array}$ & 12-60 months & $\begin{array}{l}\text { BCG, } 3 \text { doses of DPT/OPV } \\
\text { and measles }\end{array}$ & $\begin{array}{l}\text { sprimary; } \\
\text { >primary }\end{array}$ & Weak \\
\hline $\begin{array}{l}\text { Luman et al. } \\
2003[38]\end{array}$ & USA & National & Cross-sectional & $\begin{array}{l}\text { July } 2000- \\
\text { June } 2001\end{array}$ & 21,212 children & $\begin{array}{l}19 \text { to } \\
35 \text { months }\end{array}$ & $\begin{array}{l}4 \text { doses of DPT vaccine, } \\
3 \text { doses of poliovirus vaccine, } \\
1 \text { dose of MMR vaccine, } \\
3 \text { or } 4 \text { doses of Hib vaccine, } \\
\text { and } 3 \text { doses of HBV vaccine } \\
\text { (the 4:3:1:3:3 series). }\end{array}$ & $\begin{array}{l}\text { <High school; } \\
\text { High school; } \\
\text { >High school; } \\
\text { College graduate }\end{array}$ & Moderate \\
\hline $\begin{array}{l}\text { Mohamud et al. } \\
2014 \text { [39] }\end{array}$ & Ethiopia & Region & Cross-sectional & $\begin{array}{l}10 \text { April 2011- } \\
5 \text { May } 2011\end{array}$ & 582 households & $12-23$ months & $\begin{array}{l}1 \text { dose BCG, } 1 \text { dose Measles, } \\
3 \text { doses pent/OPV before } \\
1 \text { year of age }\end{array}$ & Illiterate; Literate & Moderate \\
\hline $\begin{array}{l}\text { Odusanya et al. } \\
2008 \text { [40] }\end{array}$ & Nigeria & Rural & Cross-sectional & $\begin{array}{l}\text { September } \\
2006\end{array}$ & $\begin{array}{l}339 \text { mothers and } \\
\text { children }\end{array}$ & 12-23 months & $\begin{array}{l}\text { BCG, } 3 \text { doses of OPV \& } \\
\text { DTP, } 3 \text { doses of HBV } \\
\text { and measles vaccine }\end{array}$ & $\begin{array}{l}\text { None/ primary; } \\
\text { Secondary/ } \\
\text { university }\end{array}$ & Moderate \\
\hline $\begin{array}{l}\text { Okoro et al. } \\
2014[41]\end{array}$ & Nigeria & Region & Cross-sectional & $\begin{array}{l}\text { May to } \\
\text { December }\end{array}$ & 168 children & $\begin{array}{l}6 \text { months - } \\
5 \text { years }\end{array}$ & $\begin{array}{l}\text { Full schedule } \\
\text { (not specified) }\end{array}$ & $\begin{array}{l}\text { No formal } \\
\text { education; Primary; } \\
\text { Secondary; } \\
\text { Post-secondary; } \\
\text { University }\end{array}$ & Moderate \\
\hline $\begin{array}{l}\text { Pati et al. } \\
2011 \text { [42] }\end{array}$ & USA & Urban & Cohort & $\begin{array}{l}\text { June 15th } \\
2005 \\
\text { to August 6th } \\
2006\end{array}$ & $\begin{array}{l}506 \text { Medicaid- } \\
\text { eligible mother- } \\
\text { infant dyads }\end{array}$ & $\begin{array}{l}\text { Cohort followed } \\
\text { until } 7 \text { months }\end{array}$ & $\begin{array}{l}\text { UTD at } 7 \text { months if } \\
\text { received } 3 \text { HepB, } 2 \text { polio, } \\
\text { at least } 2 \text { Hib, } 3 \text { PCV7 } \\
\text { and } 3 \text { DTaP } \\
\text { containing vaccines }\end{array}$ & $\begin{array}{l}\text { Less than high } \\
\text { school; High } \\
\text { school; More than } \\
\text { high school }\end{array}$ & Moderate \\
\hline $\begin{array}{l}\text { Phukan et al. } \\
2008 \text { [43] }\end{array}$ & India & Region & Cross-sectional & $\begin{array}{l}\text { June and } \\
\text { July } 2003\end{array}$ & 616 children & $12-23$ months & $6 \mathrm{EPI}$ vaccines in time & $\begin{array}{l}\text { Illiterate; Primary; } \\
\text { Middle; Higher }\end{array}$ & Weak \\
\hline $\begin{array}{l}\text { Robert et al. } \\
\text { 2014a [44] }\end{array}$ & Belgium & Region & Cross-sectional & 2012 & 519 children & 18-24 months & $\begin{array}{l}\text { Hexavalent, pneumococcal, } \\
\text { MMR, meningococcal C }\end{array}$ & $\begin{array}{l}\text { Maximum } \\
\text { secondary level; } \\
\text { Higher than } \\
\text { secondary level }\end{array}$ & Moderate \\
\hline $\begin{array}{l}\text { Robert et al. } \\
2014 b[44]\end{array}$ & Belgium & Region & Cross-sectional & 2012 & 538 children & 18-24 months & $\begin{array}{l}\text { Hexavalent, pneumococcal, } \\
\text { MMR, meningococcal C }\end{array}$ & $\begin{array}{l}\text { Maximum } \\
\text { secondary level; } \\
\text { Higher than } \\
\text { secondary level }\end{array}$ & \\
\hline $\begin{array}{l}\text { Rossi et al. } \\
2015 \text { [45] }\end{array}$ & Zimbabwe & National & Cross-sectional & 2010-2011 & 1031 children & $12-23$ months & $\begin{array}{l}1 \text { dose } B C G, 1 \text { dose } \\
\text { Measles, } 3 \text { doses of Polio, } \\
3 \text { doses DPT/Pentavalent }\end{array}$ & $\begin{array}{l}\text { No education or } \\
\text { primary; Secondary } \\
\text { or higher }\end{array}$ & Moderate \\
\hline $\begin{array}{l}\text { Schoeps et al. } \\
2013 \text { [46] }\end{array}$ & $\begin{array}{l}\text { Burkina } \\
\text { Faso }\end{array}$ & Region & Cross-sectional & $\begin{array}{l}\text { September } \\
2008- \\
\text { December } \\
2009\end{array}$ & 1665 children & 12-23 months & $\begin{array}{l}\text { BCG, Oral Polio, } \\
\text { Pentavalent, yellow fever, } \\
\text { measles }\end{array}$ & Any; None & Moderate \\
\hline $\begin{array}{l}\text { Setse et al. } \\
2006[47]\end{array}$ & Zambia & $\begin{array}{l}\text { Hospital/ } \\
\text { Urban }\end{array}$ & Cross-sectional & $\begin{array}{l}\text { January } 1998 \\
\text { and October } \\
2000\end{array}$ & $\begin{array}{l}473 \text { children } \\
\text { hospitalised with } \\
\text { measles- } 372 \text { in } \\
\text { subgroup analysis }\end{array}$ & $\begin{array}{l}4 \text { and } \\
60 \text { months }\end{array}$ & $\begin{array}{l}\text { BCG and completed } \\
\text { the series of DTP } \\
\text { and OPV vaccines. }\end{array}$ & $\begin{array}{l}\text { Less than } 7 \text { years; } \\
7 \text { years; Greater } \\
\text { than } 7 \text { years }\end{array}$ & Moderate \\
\hline
\end{tabular}


Table 1 Study characteristics (Continued)

\begin{tabular}{|c|c|c|c|c|c|c|c|c|c|}
\hline Reference & Country & $\begin{array}{l}\text { Study } \\
\text { setting }\end{array}$ & $\begin{array}{l}\text { Study } \\
\text { design }\end{array}$ & $\begin{array}{l}\text { Study } \\
\text { period }\end{array}$ & Population & $\begin{array}{l}\text { Children's } \\
\text { age }\end{array}$ & Vaccine type & $\begin{array}{l}\text { Maternal } \\
\text { education } \\
\text { parameter }\end{array}$ & Quality \\
\hline $\begin{array}{l}\text { Sia et al. } \\
2009[5]\end{array}$ & $\begin{array}{l}\text { Burkina } \\
\text { Faso }\end{array}$ & Rural & Cross-sectional & 1998 & 805 children & 12-23 months & $\begin{array}{l}\text { BCG, measles, yellow fever } \\
\text { vaccines and } 3 \text { doses of } \\
\text { DTP and OPV }\end{array}$ & $\begin{array}{l}\text { No schooling; } \\
\text { Primary or } \\
\text { secondary school }\end{array}$ & Moderate \\
\hline $\begin{array}{l}\text { Singh et al. } \\
2000 \text { [48] }\end{array}$ & India & National & Cross-sectional & $\begin{array}{l}\text { June-October } \\
1999\end{array}$ & 18,783 children & 12-23 months & BCG, DPT, OPV, Measles & $\begin{array}{l}\text { Illiterate; Primary; } \\
\text { Middle; Higher } \\
\text { secondary; } \\
\text { Graduate }\end{array}$ & Weak \\
\hline $\begin{array}{l}\text { Singh et al. } \\
2001 \text { [49] }\end{array}$ & India & Region & Cross-sectional & $\begin{array}{l}\text { June-October } \\
1999\end{array}$ & 6171 children & 12-32 months & BCG, DPT, OPV, Measles & $\begin{array}{l}\text { Illiterate; Primary } \\
\text { Middle; Higher } \\
\text { secondary; } \\
\text { Graduate }\end{array}$ & Weak \\
\hline $\begin{array}{l}\text { Som et al. } \\
2010 \text { [50] }\end{array}$ & India & Region & Cross-sectional & 2002 to 2004 & 1279 children & 12-35 months & $\begin{array}{l}\text { BCG, } 3 \text { injections of DPT, } 3 \\
\text { doses of polio (excluding } \\
\text { polio 0) and } 1 \text { of measles }\end{array}$ & $\begin{array}{l}\text { Can't read and } \\
\text { write; Can read } \\
\text { and write }\end{array}$ & Moderate \\
\hline $\begin{array}{l}\text { Streatfield et al. } \\
1990 \text { [51] }\end{array}$ & Indonesia & Rural & Cross-sectional & 1989 & $\begin{array}{l}519 \text { mother-child } \\
\text { dyads }\end{array}$ & $\begin{array}{l}\text { Under the age } \\
\text { of } 5 \text { years }\end{array}$ & DPT, BCG, and anti-polio & $\begin{array}{l}\text { Not literate; Some } \\
\text { primary; Complete } \\
\text { primary; Secondary } \\
\text { school }\end{array}$ & Weak \\
\hline $\begin{array}{l}\text { Thang et al. } \\
2007 \text { [52] }\end{array}$ & Vietnam & National & Cross-sectional & 2002 & 468 children & 11-23 months & $\begin{array}{l}\text { BCG vaccination } 3 \text { doses of } \\
\text { DPT vaccine; at least } 3 \\
\text { doses of polio vaccine; and } \\
1 \text { dose of measles vaccine }\end{array}$ & $\begin{array}{l}\text { Illiterate; } \\
\text { Lower primary; } \\
\text { Completed } \\
\text { primary; } \\
\text { Completed } \\
\text { secondary; } \\
\text { Completed } \\
\text { high school + }\end{array}$ & Moderate \\
\hline $\begin{array}{l}\text { Torun et al. } \\
2006 \text { [53] }\end{array}$ & Turkey & Region & Cross-sectional & 2005 & $\begin{array}{l}\text { Parents of } 221 \\
\text { children }\end{array}$ & $\begin{array}{l}9 \text { month- } \\
6 \text { years of age }\end{array}$ & $\begin{array}{l}<18 \text { months completely } \\
\text { vaccinated if had } 1 \text { dose of } \\
\text { BCG, } 3 \text { doses of HBV, OPV } \\
\text { and DPT and } 1 \text { dose of } \\
\text { Measles vaccine. } \\
>18 \text { months completely } \\
\text { vaccinated if had booster } \\
\text { doses for OPV and } \\
\text { DPT vaccines }\end{array}$ & $\begin{array}{l}\text { Illiterate; } \\
\text { Graduated primary } \\
\text { school; Graduated } \\
\text { secondary school } \\
\text { or higher } \\
\text { education }\end{array}$ & Moderate \\
\hline $\begin{array}{l}\text { Waters et al. } \\
\text { 2004a [54] }\end{array}$ & Cameroon & National & Cross-sectional & 1998 & 2123 children & $\begin{array}{l}\text { Younger than } \\
3 \text { years }\end{array}$ & $\begin{array}{l}\text { By } 6 \text { weeks- } 1 \text { st dose of } \\
\text { DPT and the } 2 \text { nd dose of } \\
\text { polio vaccine; } \\
\text { By } 10 \text { weeks- } 2 \text { nd dose of } \\
\text { DPT and the } 3 \text { rd dose of } \\
\text { polio vaccine: }\end{array}$ & $\begin{array}{l}\text { Less than primary } \\
\text { school; Primary } \\
\text { school; Secondary } \\
\text { education; Higher } \\
\text { education }\end{array}$ & Moderate \\
\hline $\begin{array}{l}\text { Waters et al. } \\
\text { 2004b [54] }\end{array}$ & Cameroon & National & Cross-sectional & 2000 & 3582 children & $\begin{array}{l}\text { Younger than } \\
5 \text { years }\end{array}$ & $\begin{array}{l}\text { By } 14 \text { weeks- } 3 \text { rd DPT } \\
\text { dose; } \\
\text { By } 9 \text { months- measles vaccine }\end{array}$ & $\begin{array}{l}\text { Less than primary } \\
\text { school; } \\
\text { Primary school } \\
\text { Secondary } \\
\text { education or } \\
\text { higher education }\end{array}$ & \\
\hline $\begin{array}{l}\text { Yadav et al. } \\
2004 \text { [55] }\end{array}$ & India & Regional & Cross-sectional & $\begin{array}{l}\text { June-October } \\
1999\end{array}$ & 1481 children & 12-23 months & BCG, DPT3, OPV3, Measles & $\begin{array}{l}\text { Illiterate; Primary; } \\
\text { Middle; Hr. } \\
\text { Secondary; } \\
\text { Graduate }\end{array}$ & Weak \\
\hline
\end{tabular}

Abbreviations: UTD up to date, EPI Expanded Program on Immunization, OPV oral polio vaccine, $B C G$ bacille Calmette-Guérin (tuberculosis) vaccine, DPT diphtheria, pertussis, tetanus vaccine, Hib haemophilus influenzae type $b, H B V$ hepatitis B virus, MMR measles, mumps \& rubella vaccine, DT diphtheria and tetanus, $P C V 7$ pneumococcal conjugate vaccine (7-valent), DTaP diphtheria, tetanus and acellular pertussis vaccine, DTCP diphtheria, tetanus, pertussis, poliomyelitis vaccine

compared both. Many were population based studies, and two were conducted in a hospital setting.

Full details of the included articles are presented in Table 1 showing the characteristics of the papers included and the quality of the studies that were compared. The majority (26 studies) were of moderate quality, with only one found to be of strong quality. Ten studies scored a global score of weak but were still included in the analysis due to the small number of studies available. Most of the studies were well conducted, but their cross-sectional study design meant the global score was brought down. The sample size ranged from 220 households (with 110 children) to 21,212 children in a cross-sectional American study. The total number of children was 112,841 , with a mean of 836 children and median of 190 children per study (calculated from Table 2). Of the 33 included in the meta-analysis, the total number of children was 92,192, with a mean of 2794 and a median of 693 . The age range was from birth to seven years, with the majority of studies using 12-23 months as the objective population due to 
Table 2 Study results

\begin{tabular}{|c|c|c|c|c|c|}
\hline Reference & $\begin{array}{l}\text { Maternal education } \\
\text { parameter }\end{array}$ & $\begin{array}{l}\text { \# children whose } \\
\text { mothers had } \\
\text { education level }\end{array}$ & $\begin{array}{l}\text { \# children who have received } \\
\text { full vaccination schedule }\end{array}$ & $\begin{array}{l}\text { \% children who received } \\
\text { full vaccination schedule } \\
\text { (1 d.p.) }\end{array}$ & $\begin{array}{l}\text { cOR for vaccination } \\
\text { (2 d.p.) }\end{array}$ \\
\hline \multirow{8}{*}{$\begin{array}{l}\text { Al-Sheikh et al. } \\
\text { 1999a [17] }\end{array}$} & Illiterate & 27 & 22 & 81.5 & 1 \\
\hline & Reads and writes & 69 & 41 & 59.4 & 0.33 \\
\hline & Primary & 78 & 42 & 53.8 & 0.27 \\
\hline & Intermediate & 32 & 22 & 68.8 & 0.5 \\
\hline & Secondary & 53 & 29 & 54.7 & 0.27 \\
\hline & Institute & 43 & 27 & 62.8 & 0.38 \\
\hline & College & 23 & 12 & 52.2 & 0.25 \\
\hline & Postgraduate & 1 & 1 & 100 & / \\
\hline \multirow{8}{*}{$\begin{array}{l}\text { Al-Sheikh et al. } \\
\text { 1999b [17] }\end{array}$} & Illiterate & 143 & 34 & 23.8 & 1 \\
\hline & Reads and writes & 121 & 34 & 28.1 & 1.25 \\
\hline & Primary & 50 & 10 & 20 & 0.8 \\
\hline & Intermediate & 5 & 2 & 40 & 2.14 \\
\hline & Secondary & 7 & 5 & 71.4 & 8.01 \\
\hline & Institute & 6 & 4 & 66.7 & 6.41 \\
\hline & College & 4 & 4 & 100 & / \\
\hline & Postgraduate & 0 & / & / & / \\
\hline \multirow{3}{*}{$\begin{array}{l}\text { Animaw et al. } \\
2014 \text { [24] }\end{array}$} & None & 262 & 150 & 57.3 & 1.00 \\
\hline & Primary & 252 & 211 & 83.8 & 3.84 \\
\hline & High school & 116 & 100 & 86.2 & 4.66 \\
\hline \multirow[t]{3}{*}{ Antai 2009 [4] } & No education & 2155 & 169 & 7.8 & 1 \\
\hline & Primary & 805 & 142 & 17.6 & 2.52 \\
\hline & Secondary or higher & 771 & 194 & 25.2 & 3.95 \\
\hline \multirow[t]{3}{*}{ Antai 2012 [20] } & No education & 12,265 & 722 & 5.9 & 1 \\
\hline & Primary school & 5724 & 1159 & 20.2 & 4.06 \\
\hline & Secondary school or higher & 6921 & 2402 & 34.7 & 8.50 \\
\hline \multirow{4}{*}{$\begin{array}{l}\text { Bbaale et al. } \\
2013 \text { [25] }\end{array}$} & No education & 1824 & 967 & 53.0 & 1.00 \\
\hline & Primary & 4686 & 2484 & 53.0 & 1.00 \\
\hline & Secondary & 896 & 520 & 58.0 & 1.23 \\
\hline & Post-secondary & 185 & 117 & 63.2 & 1.52 \\
\hline \multirow{2}{*}{$\begin{array}{l}\text { Branco et al. } \\
2014 \text { [26] }\end{array}$} & $0-8$ years of schooling & 151 & 116 & 76.8 & 1.00 \\
\hline & $>8$ years of schooling & 130 & 117 & 90 & 2.72 \\
\hline \multirow{2}{*}{$\begin{array}{l}\text { Brenner et al. } \\
2001 \text { [27] }\end{array}$} & $<12$ years & 145 & $55 a$ & 38 & 1 \\
\hline & $\geq 12$ years & 179 & $77 \mathrm{a}$ & 43 & 1.23 \\
\hline \multirow{2}{*}{$\begin{array}{l}\text { Calhoun et al. } \\
2014 \text { [28] }\end{array}$} & $0-7$ years of schooling & 132 & 35 & 26.5 & 1.00 \\
\hline & $\geq 8$ years of schooling & 23 & 11 & 47.8 & 2.54 \\
\hline \multirow{3}{*}{$\begin{array}{l}\text { Chhabra et al. } \\
2007 \text { [29] }\end{array}$} & Nil & $378_{b}$ & 130 & 34.4 & 1 \\
\hline & $1-8$ years & $106_{b}$ & 51 & 48.1 & 1.77 \\
\hline & $>8$ years & $209_{b}$ & 106 & 50.7 & 1.96 \\
\hline \multirow{5}{*}{$\begin{array}{l}\text { Danis et al. } \\
2010 \text { [18] }\end{array}$} & $<9$ years & 536 & 278 & 51.9 & 1 \\
\hline & $9-11$ years & 429 & 240 & 55.9 & 1.18 \\
\hline & 12 years (high school) & 1336 & 859 & 64.3 & 1.67 \\
\hline & College/ university graduate & 985 & 670 & 68 & 1.97 \\
\hline & Illiterate & $332_{b}$ & 240 & 72.3 & 1 \\
\hline
\end{tabular}


Table 2 Study results (Continued)

\begin{tabular}{|c|c|c|c|c|c|}
\hline Reference & $\begin{array}{l}\text { Maternal education } \\
\text { parameter }\end{array}$ & $\begin{array}{l}\text { \# children whose } \\
\text { mothers had } \\
\text { education level }\end{array}$ & $\begin{array}{l}\text { \# children who have received } \\
\text { full vaccination schedule }\end{array}$ & $\begin{array}{l}\text { \% children who received } \\
\text { full vaccination schedule } \\
\text { (1 d.p.) }\end{array}$ & $\begin{array}{l}\text { COR for vaccination } \\
\text { ( } 2 \text { d.p.) }\end{array}$ \\
\hline $\begin{array}{l}\text { Elliott et al. } \\
\text { 2006a [30] }\end{array}$ & Literate & $139_{b}$ & 123 & 88.5 & 2.95 \\
\hline \multirow{2}{*}{$\begin{array}{l}\text { Elliott et al. } \\
\text { 2006b [30] }\end{array}$} & Illiterate & $318_{b}$ & 210 & 66 & 1 \\
\hline & Literate & $127_{b}$ & 113 & 89 & 4.15 \\
\hline \multirow{2}{*}{$\begin{array}{l}\text { Elliott et al. } \\
2006 c \text { [30] }\end{array}$} & Illiterate & $139 b$ & 73 & 52.5 & 1 \\
\hline & Literate & $49_{b}$ & 35 & 71.4 & 2.26 \\
\hline \multirow{2}{*}{$\begin{array}{l}\text { Fatiregun et al. } \\
2012 \text { [31] }\end{array}$} & Primary/ secondary & 297 & 76 & 25.6 & 1 \\
\hline & Post secondary & 228 & 94 & 41.2 & 2.04 \\
\hline \multirow{4}{*}{$\begin{array}{l}\text { Fatiregun et al. } \\
2013 \text { [32] }\end{array}$} & None & 129 & 24 & 18.6 & 1.00 \\
\hline & Primary & 468 & 128 & 27.4 & 1.65 \\
\hline & Secondary & 523 & 225 & 43.0 & 3.30 \\
\hline & Tertiary & 58 & 51 & 87.9 & 31.88 \\
\hline \multirow{3}{*}{$\begin{array}{l}\text { Huq et al. } \\
2008 \text { [33] }\end{array}$} & Below primary & 485 & $307 a$ & 63.3 & 1 \\
\hline & Secondary & 221 & $164_{a}$ & 74.2 & 1.67 \\
\hline & Higher secondary & 49 & $46_{a}$ & 93.9 & 8.92 \\
\hline \multirow{3}{*}{$\begin{array}{l}\text { Jahn et al. } \\
2008 \text { [34] }\end{array}$} & $<5$ years primary & 237 & 140 & 59.1 & 1 \\
\hline & Primary $5+$ years & 1364 & 903 & 66.2 & 1.36 \\
\hline & Sec./tert. & 304 & 233 & 76.6 & 2.27 \\
\hline \multirow{2}{*}{$\begin{array}{l}\text { Kidane et al. } \\
2003 \text { [35] }\end{array}$} & Illiterate & 92 & 66 & 71.7 & 1 \\
\hline & Literate & 18 & 17 & 94.4 & 6.70 \\
\hline \multirow{2}{*}{$\begin{array}{l}\text { Koumaré et al. } \\
2009 \text { [36] }\end{array}$} & Mother not educated & 639 & $376_{a}$ & 58.8 & 1 \\
\hline & Mother educated & 111 & $73 a$ & 65.8 & 1.35 \\
\hline \multirow{2}{*}{$\begin{array}{l}\text { Kumar et al. } \\
2010 \text { [37] }\end{array}$} & $\leq$ primary & 223 & 12 & 5.4 & 1 \\
\hline & >primary & 92 & 46 & 50.0 & 17.58 \\
\hline \multirow{4}{*}{$\begin{array}{l}\text { Luman et al. } \\
2003[38]\end{array}$} & $<$ High school & 3157 & $2147 a$ & 68.0 & 1 \\
\hline & High school & 7160 & $5191_{a}$ & 72.5 & 1.24 \\
\hline & >High school & 4375 & $3233 a$ & 73.9 & 1.33 \\
\hline & College graduate & 8698 & $6915 a$ & 79.5 & 1.82 \\
\hline \multirow{2}{*}{$\begin{array}{l}\text { Mohamud et al. } \\
2014 \text { [39] }\end{array}$} & Illiterate & 510 & 167 & 32.7 & 1.00 \\
\hline & Litterate & 72 & 46 & 63.9 & 3.63 \\
\hline \multirow{2}{*}{$\begin{array}{l}\text { Odusanya et al. } \\
2008 \text { [40] }\end{array}$} & None/ primary & 107 & 57 & 53.3 & 1 \\
\hline & Secondary/ university & 232 & 153 & 65.9 & 1.70 \\
\hline \multirow{5}{*}{$\begin{array}{l}\text { Okoro et al. } \\
2014 \text { [41] }\end{array}$} & No formal education & 12 & 7 & 58.3 & 1.00 \\
\hline & Primary & 33 & 16 & 48.5 & 0.67 \\
\hline & Secondary & 55 & 36 & 65.5 & 1.35 \\
\hline & Post-secondary & 28 & 24 & 85.7 & 4.29 \\
\hline & University & 40 & 32 & 80.0 & 2.86 \\
\hline \multirow{3}{*}{$\begin{array}{l}\text { Pati et al. } 2011 \\
\text { [42] }\end{array}$} & Less than high school & 159 & 63 & 39.6 & 1 \\
\hline & High school & 119 & 55 & 46.2 & 1.31 \\
\hline & More than high school & 228 & 101 & 44.3 & 1.21 \\
\hline \multirow{3}{*}{$\begin{array}{l}\text { Phukan et al. } \\
2008 \text { [43] }\end{array}$} & Illiterate & 132 & 50 & 37.9 & 1 \\
\hline & Primary & 81 & 41 & 50.6 & 1.68 \\
\hline & Middle & 344 & 242 & 70.3 & 3.89 \\
\hline
\end{tabular}


Table 2 Study results (Continued)

\begin{tabular}{|c|c|c|c|c|c|}
\hline Reference & $\begin{array}{l}\text { Maternal education } \\
\text { parameter }\end{array}$ & $\begin{array}{l}\text { \# children whose } \\
\text { mothers had } \\
\text { education level }\end{array}$ & $\begin{array}{l}\text { \# children who have received } \\
\text { full vaccination schedule }\end{array}$ & $\begin{array}{l}\% \text { children who received } \\
\text { full vaccination schedule } \\
\text { (1 d.p.) }\end{array}$ & $\begin{array}{l}\text { cOR for vaccination } \\
\text { ( } 2 \text { d.p.) }\end{array}$ \\
\hline & Higher & 59 & 50 & 84.7 & 9.11 \\
\hline \multirow{2}{*}{$\begin{array}{l}\text { Robert et al. } \\
\text { 2014a [44] }\end{array}$} & Maximum secondary level & 293 & 237 & 80.8 & 1.00 \\
\hline & Higher than secondary level & 214 & 177 & 82.9 & 1.13 \\
\hline \multirow{2}{*}{$\begin{array}{l}\text { Robert et al. } \\
\text { 2014b [44] }\end{array}$} & Maximum secondary level & 296 & 242 & 81.6 & 1.06 \\
\hline & Higher than secondary level & 233 & 197 & 84.4 & 1.29 \\
\hline \multirow{2}{*}{$\begin{array}{l}\text { Rossi et al. } 2015 \\
\text { [45] }\end{array}$} & No education or primary & 320 & 177 & 55.2 & 1.00 \\
\hline & Secondary or higher & 711 & 500 & 70.3 & 1.91 \\
\hline \multirow{2}{*}{$\begin{array}{l}\text { Schoeps et al. } \\
2013 \text { [46] }\end{array}$} & None & 1435 & 250 & 17.4 & 1.00 \\
\hline & Any & 230 & 57 & 24.8 & 1.56 \\
\hline \multirow{3}{*}{$\begin{array}{l}\text { Setse et al. } 2006 \\
\text { [47] }\end{array}$} & Less than 7 years & 137 & $92 a_{a}$ & $67 c$ & 1 \\
\hline & 7 years & 114 & $87 a$ & $766_{c}$ & 1.56 \\
\hline & Greater than 7 years & 121 & $105 a$ & $87 c$ & 3.30 \\
\hline \multirow{2}{*}{$\begin{array}{l}\text { Sia et al. } 2009 \\
{[5]}\end{array}$} & No schooling & 850 & $172_{a}$ & 20.2 & 1 \\
\hline & Primary or secondary school & 48 & $18 \mathrm{a}$ & 37.5 & 2.37 \\
\hline \multirow{5}{*}{$\begin{array}{l}\text { Singh et al. } 2000 \\
\text { [48] }\end{array}$} & Illiterate & 7337 & $3404_{a}$ & 46.4 & 1 \\
\hline & Primary & 2946 & $1912_{a}$ & 64.9 & 2.14 \\
\hline & Middle & 3044 & $2143_{a}$ & 70.4 & 2.75 \\
\hline & Higher secondary & 3433 & $2705_{a}$ & 78.8 & 4.29 \\
\hline & Graduate & 2023 & $1705_{a}$ & 84.3 & 6.20 \\
\hline \multirow{5}{*}{$\begin{array}{l}\text { Singh et al. } 2001 \\
\text { [49] }\end{array}$} & Illiterate & 3421 & $1143_{a}$ & 33.4 & 1 \\
\hline & Primary & 900 & $496 a$ & 55.1 & 2.45 \\
\hline & Middle & 718 & $442 a_{a}$ & 61.5 & 3.19 \\
\hline & Higher secondary & 580 & $41 \sigma_{a}$ & 71.8 & 5.08 \\
\hline & Graduate & 552 & $442_{a}$ & 80 & 7.98 \\
\hline \multirow{2}{*}{$\begin{array}{l}\text { Som et al. } 2010 \\
{[50]}\end{array}$} & Can't read and write & 400 & $151_{a}$ & 37.8 & 1 \\
\hline & Can read and write & 879 & $538_{a}$ & 61.2 & 2.60 \\
\hline \multirow{4}{*}{$\begin{array}{l}\text { Streatfield et al. } \\
1990 \text { [51] }\end{array}$} & Not literate & 78 & $35 a$ & 45.1 & 1 \\
\hline & Some primary & 129 & $40_{a}$ & 31.1 & 0.55 \\
\hline & Complete primary & 177 & $59 a$ & 33.6 & 0.62 \\
\hline & Secondary school & 81 & $44_{a}$ & 54.9 & 1.48 \\
\hline \multirow{5}{*}{$\begin{array}{l}\text { Thang et al. } \\
2007 \text { [52] }\end{array}$} & Illiterate & 33 & $13 a$ & 39.5 & 1 \\
\hline & Lower primary & 74 & $37 a$ & 50 & 1.53 \\
\hline & Completed primary & 157 & $100_{a}$ & 63.5 & 2.66 \\
\hline & Completed secondary & 122 & $94 a$ & 77.4 & 5.25 \\
\hline & Completed high school + & 83 & 69 a & 82.9 & 7.43 \\
\hline \multirow{3}{*}{$\begin{array}{l}\text { Torun et al. } 2006 \\
\text { [53] }\end{array}$} & Illiterate & $31_{b}$ & 15 & 48.4 & 1 \\
\hline & Graduated primary school & $157_{b}$ & 141 & 89.8 & 9.4 \\
\hline & $\begin{array}{l}\text { Graduated secondary school or } \\
\text { higher education }\end{array}$ & $33_{b}$ & 31 & 93.9 & 16.53 \\
\hline \multirow{4}{*}{$\begin{array}{l}\text { Waters et al. } \\
\text { 2004a [54] }\end{array}$} & Less than primary school & 438 & $105 a$ & 24 & 1 \\
\hline & Primary school & 603 & $235 a$ & 39 & 2.02 \\
\hline & Secondary education & 473 & $246_{a}$ & 52 & 3.43 \\
\hline & Higher education & 12 & $8 a$ & 67 & 6.43 \\
\hline
\end{tabular}


Table 2 Study results (Continued)

\begin{tabular}{|c|c|c|c|c|c|}
\hline Reference & $\begin{array}{l}\text { Maternal education } \\
\text { parameter }\end{array}$ & $\begin{array}{l}\text { \# children whose } \\
\text { mothers had } \\
\text { education level }\end{array}$ & $\begin{array}{l}\text { \# children who have received } \\
\text { full vaccination schedule }\end{array}$ & $\begin{array}{l}\% \text { children who received } \\
\text { full vaccination schedule } \\
\text { (1 d.p.) }\end{array}$ & $\begin{array}{l}\text { cOR for vaccination } \\
\text { (2 d.p.) }\end{array}$ \\
\hline \multirow{3}{*}{$\begin{array}{l}\text { Waters et al. } \\
\text { 2004b [54] }\end{array}$} & Less than primary school & 961 & $202_{a}$ & 21 & 1 \\
\hline & Primary school & 1137 & $387 a$ & 34 & 1.94 \\
\hline & $\begin{array}{l}\text { Secondary education or higher } \\
\text { education }\end{array}$ & 840 & $403 a$ & 48 & 3.47 \\
\hline \multirow{5}{*}{$\begin{array}{l}\text { Yadav et al. } \\
2004 \text { [55] }\end{array}$} & Illiterate & $835 d$ & $407 a$ & 48.7 & 1 \\
\hline & Primary & 241 & $180_{a}$ & 74.8 & 3.13 \\
\hline & Middle & 190 & $142_{a}$ & 74.9 & 3.14 \\
\hline & Hr. Secondary & 119 & $93 a$ & 78.2 & 3.78 \\
\hline & Graduate & 96 & $77 a$ & 80.2 & 4.27 \\
\hline
\end{tabular}

d.p. = decimal places

a Number of children fully vaccinated calculated using available data in the paper (i.e. \% uptake x total number of children)

b Total number of children per maternal education level calculated from adding row total

c Reverse percentage calculated from data in paper (percentage incompletely vaccinated presented)

${ }_{d}$ Number of children with an illiterate mother calculated from deducting number in other levels from total population size

the EPI schedule targeting this age group [16]. The papers using demographic health survey (DHS) data were conducted on women aged 15-49 years old. On most other papers, this was not specified.

Maternal education levels varied between the study settings, with those set in higher income countries having higher baselines, potentially due to difference in schooling between countries. Dichotomous variables were used in 14 studies where the woman was classed as either literate or not, or above or below a set threshold.

\section{Data extraction}

The raw results show a general increase in vaccination completion with increasing maternal education within the separate papers (Table 2). The odd ratios between the highest and lowest education levels within the studies ranged from 0.25 , showing a decrease in completion, to 31.88 showing hugely increased odds of the children being fully vaccinated if the mother was more educated than the baseline group. Only two studies showed decreased odds between lowest and highest education levels, with the rest all showing a positive trend. Percentage fully vaccinated also varied widely from $1.0 \%$ to $100 \%$ with an average of $55.9 \%$ having completed the immunisation schedule. These variations are further explored by the meta-analysis.

\section{Meta-analysis}

Overall, the meta-analysis showed that the odds of full childhood vaccination were 2.31 times (95\% CI 1.90-2.79) greater in children whose mothers had received secondary or higher education when compared to those whose mothers had no education or primary level education (Fig. 2). Although all but four studies showed a positive effect of being highly educated, the effect size varied greatly between papers, with an overall I-squared value of 95.0\% $(p<0.001)$, indicating a high level of heterogeneity.

\section{Illiteracy vs. literacy}

Figure 3 shows a separate meta-analysis of six studies which split mothers based upon whether they were literate or illiterate. It demonstrates full vaccination of children was more likely in mothers that were literate compared to illiterate, with an odds ratio of 2.87 (95\% CI 2.39-3.46).

\section{Continent}

Subgroup analysis of continents (Fig. 4) showed the overall effect size is highest in Asia, where the odds of full childhood vaccination were 2.65 times (95\% CI 2.08-3.37) greater if the mother was more educated. Only one result out of 11 was not statistically significant (Al-Sheikh et al. 1999a) [17].

The overall effect for Africa was increased odds of 2.34 (95\% CI 1.69-3.24) for completion of childhood vaccination with higher maternal education. There were no statistically insignificant papers in this subgroup.

The overall effect was lower in the higher income continent of Europe, with increased odds of 1.47 (95\% CI 1.14-1.89) for completion of childhood vaccination with higher maternal education. Furthermore, three-quarters of European papers had statistically insignificant results, and low heterogeneity.

\section{Setting}

Within the setting subgroup analysis (Fig. 5), vaccination of children was most likely in highly educated women in rural areas, with an odds ratio 2.17 (95\% CI 1.48-3.17). There was no statistically significant difference in the odds ratios between the rural and urban settings. 


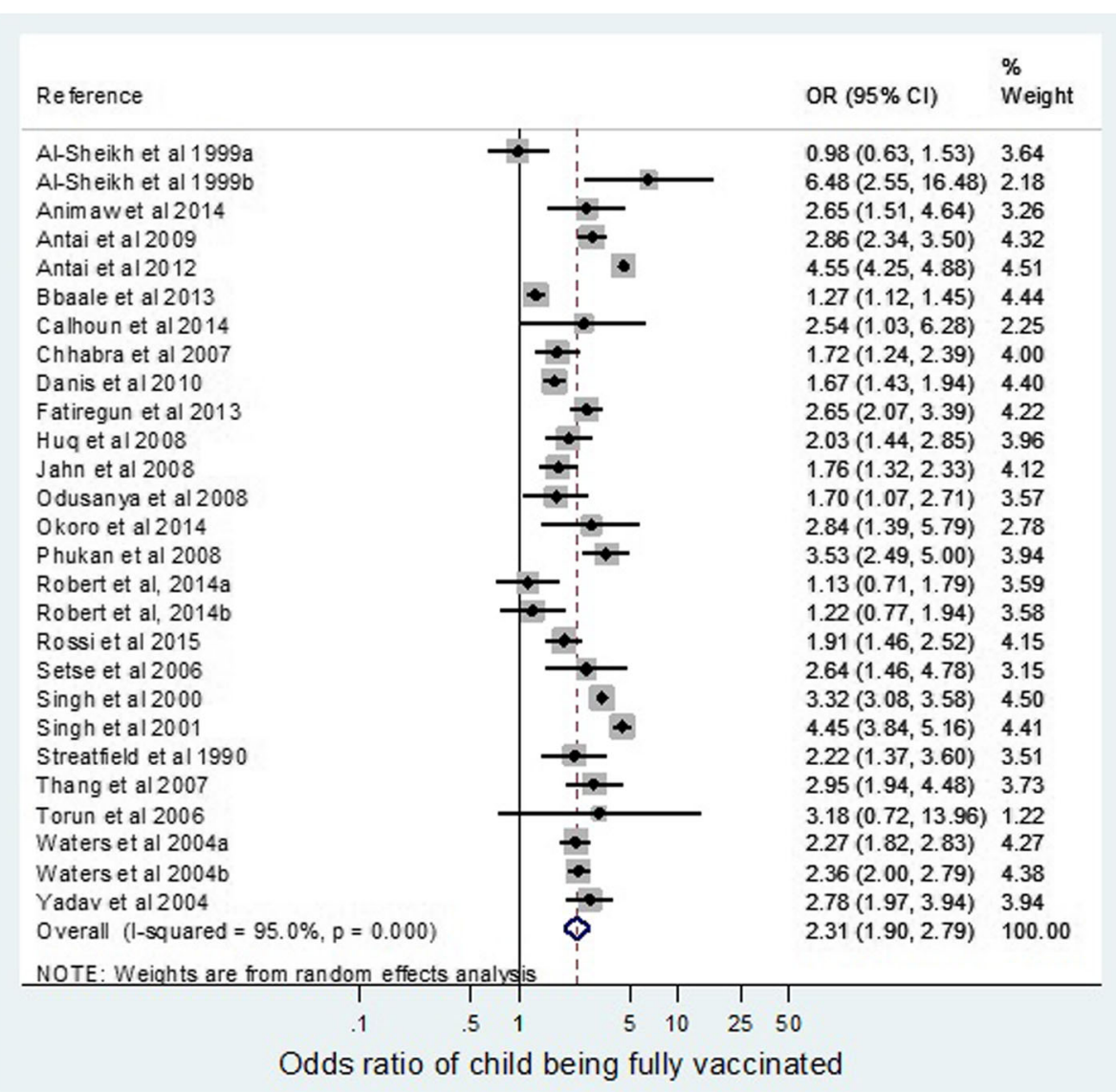

Fig. 2 Odds ratio of children being fully vaccinated if mother educated to a secondary level compared with no or primary education

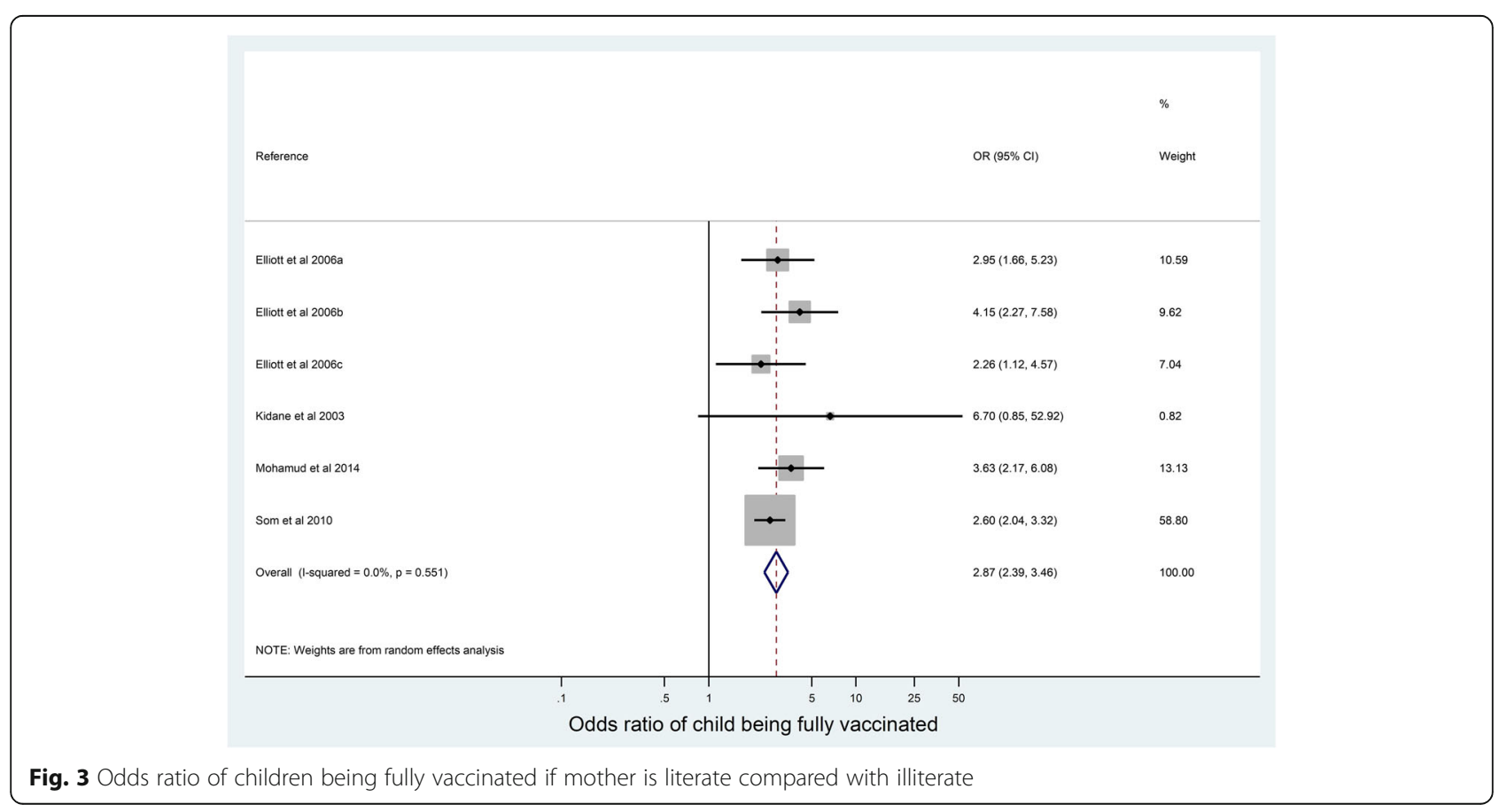




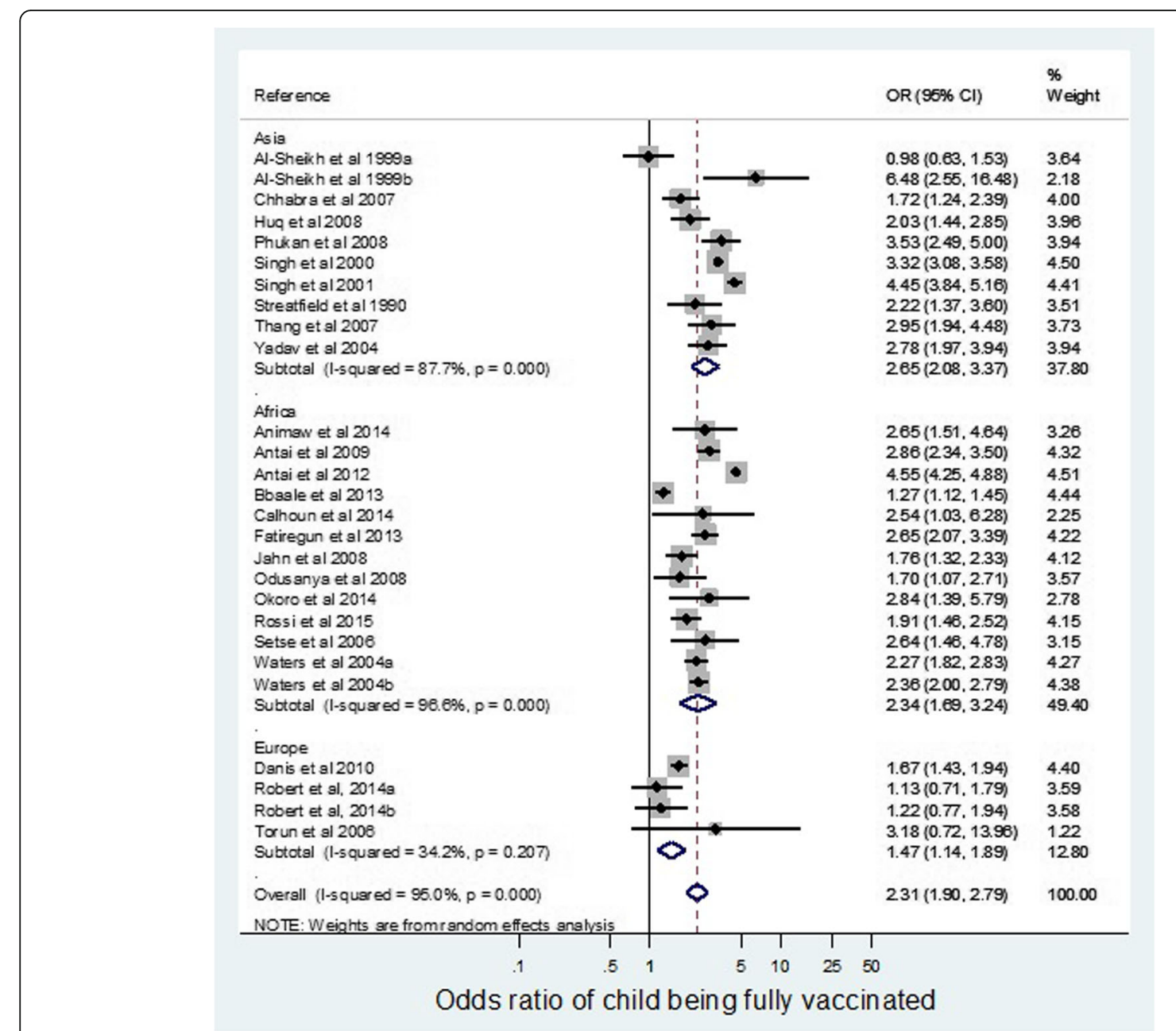

Fig. 4 Odds ratio of children being fully vaccinated if mother educated to a secondary level compared with no or primary education, according to continent

\section{Timing}

As seen in Fig. 6, studies conducted before 2000 show an odds ratio of 2.58 (95\% CI 2.04-3.26). The overall odds ratio for studies conducted from 2001 is 2.18 (95\% CI 1.62-2.94). Although the odds of complete child vaccination are slightly lower in the later time period, there was no statistically significant difference in the odds ratios.

\section{Summary estimate of vaccine completion by maternal education level}

Collapsing of the different maternal education variables into the 4 categories, none, primary, secondary or tertiary education, to obtain the pooled estimate of the percentage of children fully vaccinated per strata is shown in Table 3. This demonstrates an increase in completion of vaccination as the maternal education level increases. Only $42.8 \%$ (95\% CI 35.2-50.4) of children whose mothers had no education were fully vaccinated. This increases to 80.2\% (95\% CI 75.5-85.0) amongst children whose mothers had completed tertiary education. The pooled summary also shows that there is the overall prevalence of vaccination uptake was $57.8 \%$ (95\% CI: 52.4-63.1).

However, there is significant heterogeneity between studies, as reflected in the I-squared values. This demonstrates that maternal education is not the only determinant of vaccination uptake.

\section{Discussion}

Summary

The primary finding of this review is that an increase in maternal education is correlated with increased childhood vaccination. However, the overall effect size of maternal education on vaccination completion cannot be concluded due to heterogeneity between the studies. Summary estimates of percentage of children fully vaccinated according to the level of maternal education showed a step-wise increase in overall percentages as maternal education increased from none to tertiary. Additionally, a significant difference was shown on the meta-analysis between 


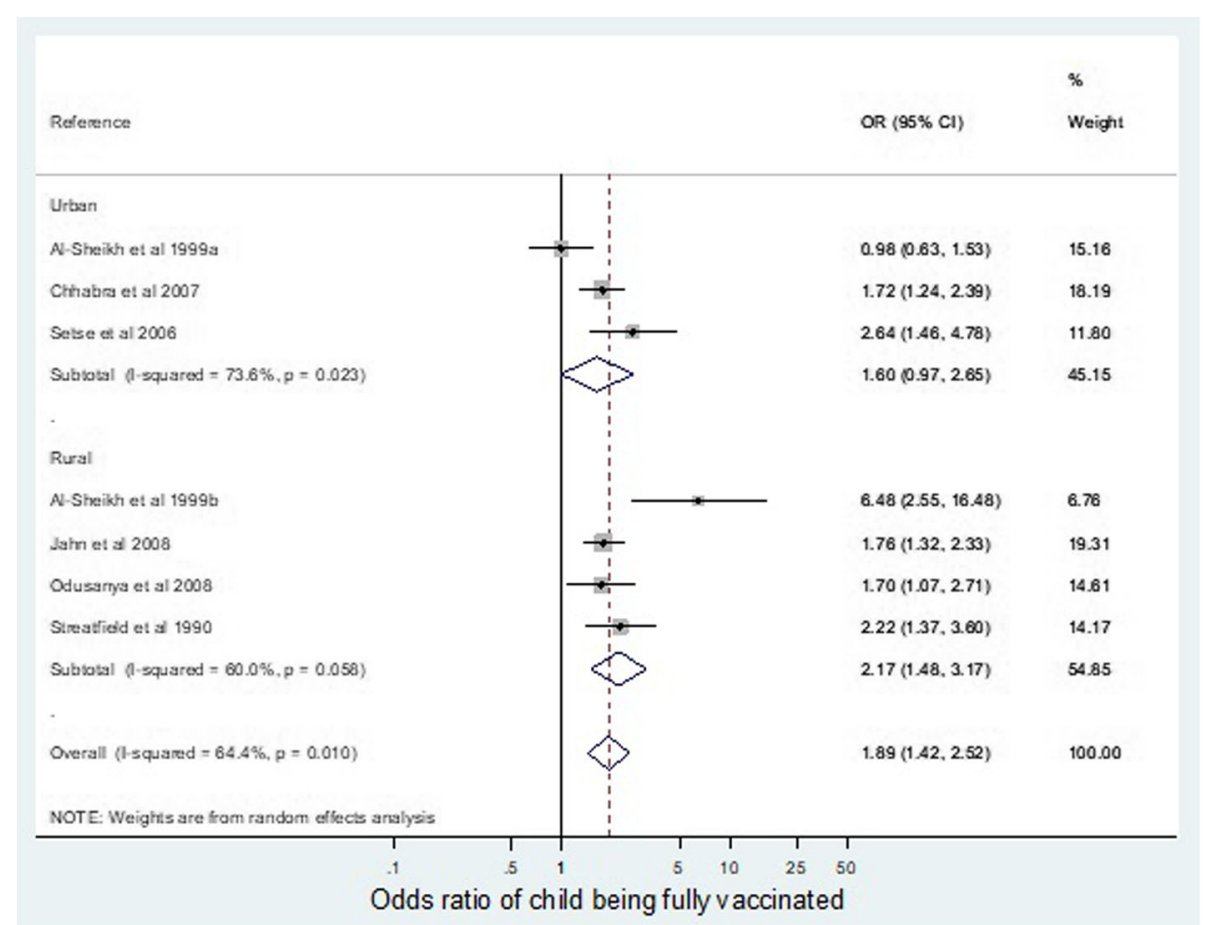

Fig. 5 odds ratio of children being fully vaccinated if mother educated to a secondary level compared with no or primary education, according to setting

literate and illiterate women, displaying that increased literacy has a beneficial impact on vaccination uptake.

This review also demonstrated a difference in the size of the effect seen between Asia and Africa compared to Europe. The higher odds ratio of maternal education on vaccination uptake in Asia and Africa may demonstrate that education plays a more important role in lower income countries. This could be due to societal development as areas with better education may also have improved healthcare access. Whilst the effect is lower in Europe, it is still positive. This demonstrates the importance of maternal education even in the presence of good health care programmes.

No difference in the effect of maternal education on vaccine uptake was found between urban and rural settings. It is of note that many of the studies were population based so are likely to be representative; however, two studies were conducted in a hospital setting so are less generalizable.

The results also show no difference in the effect of maternal education on vaccine uptake between time periods.

The heterogeneity seen between the results may be due to a number of other factors which may also affect vaccination uptake, such as availability of the immunizations, distance to healthcare facility, household income and maternal age which would confound the effect size [18]. Despite the presence of confounders, there remains a strong correlation between maternal education and child vaccination completion.

\section{Limitations}

As with all studies, this review has some limitations. The main one was the exclusion of non-English papers which could potentially lead to language bias. Moreover, authors were not contacted for the raw data if the study had been excluded due to lack of published data in the required format.

In addition, condensing the maternal education variables may have hidden subtle patterns between the smaller jumps in education level. Furthermore, this meant that in studies with dichotomous variables of educated against not, and illiterate vs literate, the educated variable was also categorised as "none/primary" in the meta-analysis. Due to the differences in the settings of the studies, there was no universal standard for measuring level of education. In order to compare them in this review, they were categorised into set variables which contributed to the high heterogeneity.

\section{Implications of this review}

This current review adds further evidence of the association between maternal education and child mortality reduction [19]. It is possible that child vaccination uptake is in fact one of the pathways for which this relationship is seen. It also shows that child vaccination uptake is not solely down to supply of vaccinations, and programs which aim to increase the dispersion of immunizations need to concentrate on these additional factors 


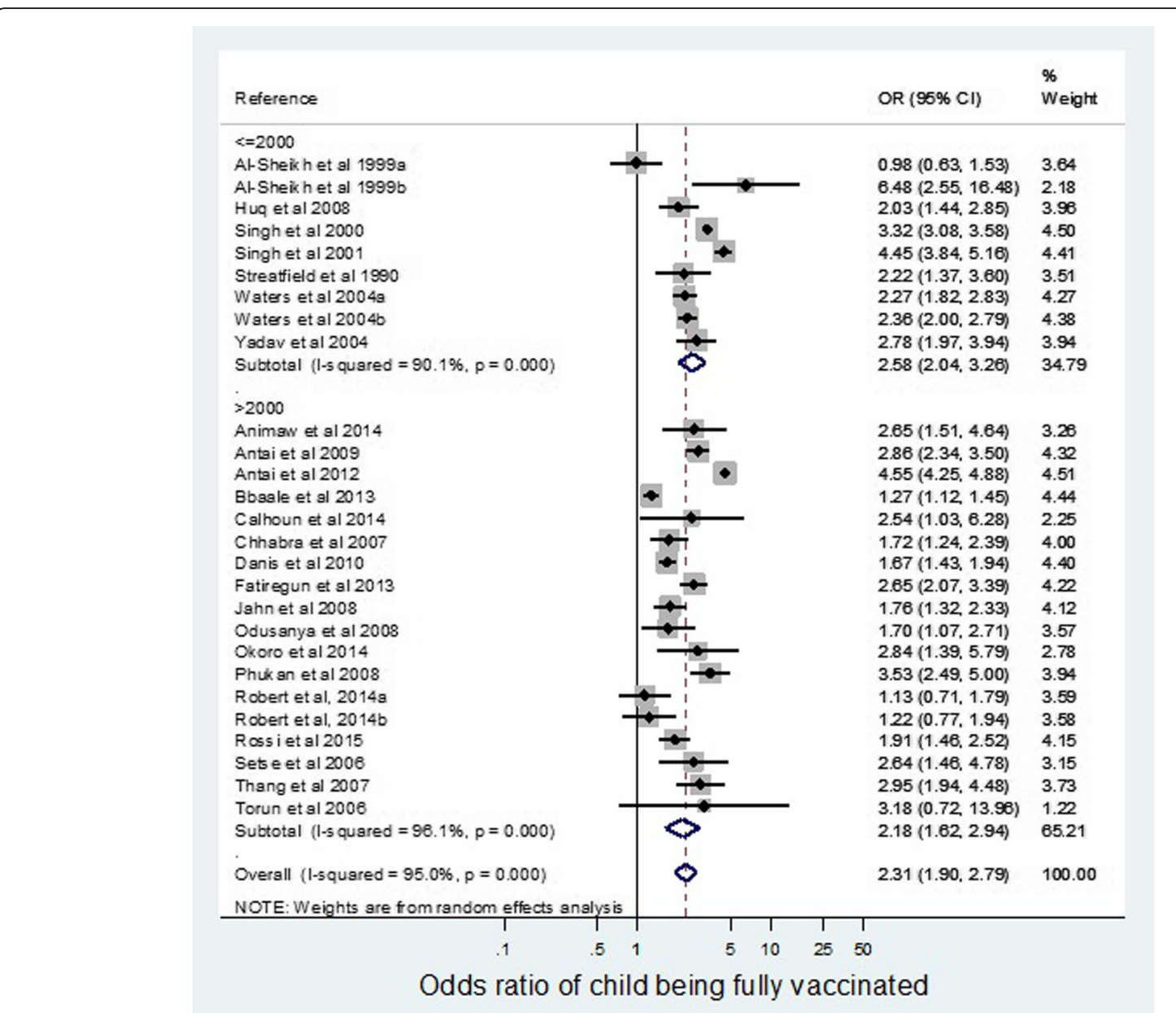

Fig. 6 Odds ratio of children being fully vaccinated if mother educated to a secondary level compared with no or primary education, according to time period

[20]. Furthermore, it adds to the current argument of the importance of educating women and gender equality [21]. Despite these associations this study does not answer the question of exactly how maternal education increases vaccine uptake. One link may be that increasing maternal education leads to more access to healthcare and therefore vaccine uptake. However, previous studies have theorised that maternal education, specifically literacy, enhance cognition and communication skills which encourage healthier lifestyle choices leading to lower childhood mortality [22].

Table 3 Pooled summary vaccination completion per education level

\begin{tabular}{llll}
\hline $\begin{array}{l}\text { Maternal } \\
\text { education level }\end{array}$ & $\begin{array}{l}\text { Pooled child vaccination } \\
\text { completion (\%) }\end{array}$ & $\begin{array}{l}\text { 95\% confidence } \\
\text { interval }\end{array}$ & $\begin{array}{l}\text { I-squared } \\
(\%)\end{array}$ \\
\hline None & 42.8 & $35.2-50.4$ & 99.7 \\
Primary & 56.6 & $49.5-63.7$ & 99.4 \\
Secondary & 64.3 & $56.1-72.5$ & 99.2 \\
Tertiary & 80.2 & $75.5-85.0$ & 89.3 \\
\hline
\end{tabular}

The meta-analysis looking at literacy levels demonstrated that one of the potential mediators between maternal education and complete vaccination was maternal literacy. This is further supported by Balogun et al. who found that mothers who were literate, regardless of their education level, were more likely to vaccinate their children [23]. This therefore implies that improving the educational standards to ensure literacy will have a greater impact on increased childhood vaccination than simply increasing the throughput of girls in education.

Overall it is clear that female education is crucial in improving child health and should be considered when policies surrounding child health are implemented. Whilst this study cannot provide an overall total effect size of maternal education on child vaccination uptake, it does demonstrate that there is a consistently positive effect. This should be taken into consideration when global health policies aiming to increase the uptake of child vaccination are applied. It also highlights the importance of female education on wider factors other than self-improvement and the economy [19]. 


\section{Conclusions}

This review highlights the positive effect of maternal education on childhood vaccination uptake across different continents, settings, and time periods.

It has been long established that childhood mortality is decreased by childhood vaccination [21]. This analysis identified that increased maternal education leads to increased childhood vaccination uptake and, in turn, will decrease childhood mortality.

\section{Acknowledgements}

Not applicable

\section{Funding}

No specific funding was obtained for this study. Logan Manikam is funded by an NIHR Doctoral Research Fellowship. Sarah Gerver was funded by an MRC Population Health Science Post-Doctoral Fellowship.

\section{Availability of data and materials}

Available on request.

\section{Authors' contributions}

JF screened articles, performed data extraction, and was a major contributor in writing the manuscript. SG performed statistical analysis of the data sets. MG and EC screened articles, performed data extraction, and were major contributors in writing the manuscript. LM and HW were involved in study design and contributors in writing the manuscript. All authors read and approved the final manuscript.

\section{Ethics approval and consent to participate}

Not applicable.

\section{Consent for publication}

Not applicable.

\section{Competing interests}

The authors declare that they have no competing interests.

\section{Publisher's Note}

Springer Nature remains neutral with regard to jurisdictional claims in published maps and institutional affiliations.

\section{Author details}

'School of Public Health, Imperial College London, South Kensington Campus, London SW7 2AZ, UK. ${ }^{2}$ St George's, University of London, Cranmer Terrace, London SW17 ORE, UK. ${ }^{3}$ UCL Great Ormond Street Institute of Child Health, 30 Guildford Street, London WC1N 1EH, UK.

Received: 21 February 2017 Accepted: 6 December 2017 Published online: 28 December 2017

\section{References}

1. World Health Organization. State of the world 's vaccines and immunization; 2009. p. 1-212

2. Vikram $K$, Vanneman $R$, Desai $S$. Linkages between maternal education and childhood immunization in India. Soc Sci Med. 2012;75(2):331-9.

3. Sullivan MC, Tegegn A, Tessema F, Galea S, Hadley C. Minding the immunization gap: family characteristics associated with completion rates in rural Ethiopia. J Community Health. 2010;35(1):53-9.

4. Antai D. Inequitable childhood immunization uptake in Nigeria: a multileve analysis of individual and contextual determinants. BMC Infect Dis. 2009;9(1):181

5. Sia D, Fournier P, Kobiané J-F, Sondo BK. Rates of coverage and determinants of complete vaccination of children in rural areas of Burkina Faso (1998-2003). BMC Public Health. 2009:9(1):416.

6. Shaikh S, Taj TM, Kazi A, Ahmed J, Fatmi Z. Coverage and predictors of vaccination among children of 1-4 years of age in a rural sub-district of Sindh. J Coll Physicians Surg Pakistan. 2010;20(12):806-10.
7. $\mathrm{WHO}$, Immunization, Vaccines and Biologicals: Estimates of disease burden and cost-effectiveness. 2015. [Online]. Available: http://www.who.int/ immunization/monitoring_surveillance/burden/estimates/en/. Accessed 22 Sept 2016.

8. Rainey JJ, Watkins M, Ryman TK, Sandhu P, Bo A, Banerjee K. Reasons related to non-vaccination and under-vaccination of children in low and middle income countries: findings from a systematic review of the published literature, 1999-2009. Vaccine. 2011;29(46):8215-21.

9. Effective public health practice project. Quality assessment tool for quantitative studies. Available from: http://www.ephpp.ca/PDF/ Quality\%20Assessment\%20Tool_2010_2.pdf. Accessed 22 Sept 2016.

10. Sanderson S, Tatt ID, Higgins JPT. Tools for assessing quality and susceptibility to bias in observational studies in epidemiology: a systematic review and annotated bibliography. Int J Epidemiol. 2007;36(3):666-76.

11. Thomas BH, Ciliska D, Dobbins M, Micucci S. A process for systematically reviewing the literature: providing the research evidence for public health nursing interventions. Worldviews Evid Based Nurs. 2004;1(3):176-84.

12. Greenhalgh T. How to read a paper: papers that summarise other papers (systematic reviews and meta-analyses). BMJ. 1997:315:672-5.

13. Adekola O. Language, literacy and learning in primary schools. Washington: World Bank; 2007. p. 5-16.

14. Dersimonian R, Laird N. Meta-analysis in clinical trials. Stat Med. 1986;188:177.

15. StataCorp, "Stata statistical software: release 13." 2013.

16. Abuya BA, Onsomu EO, Kimani JK, Moore D. Influence of maternal education on child immunization and stunting in Kenya. Matern Child Health J. 2011;15(8):1389-99.

17. Al-Sheikh OG, Al-Samarrai Jl, Al-Sumaidaie MM, Mohammad SA, Al-Dujaily AA. Immunization coverage among children born between 1989 and 1994 in Saladdin governorate, Iraq. East Mediterr Heal J. 1999;5(5):933-40.

18. Danis K, Georgakopoulou T, Stavrou T, Laggas D, Panagiotopoulos T. Socioeconomic factors play a more important role in childhood vaccination coverage than parental perceptions: a cross-sectional study in Greece. Vaccine. 2010;28(7):1861-9.

19. Gakidou E, Cowling K, Lozano R, Murray CJ. Increased educational attainment and its effect on child mortality in 175 countries between 1970 and 2009: a systematic analysis. Lancet. 2010;376(9745):959-74.

20. Antai D. Gender inequities, relationship power, and childhood immunization uptake in Nigeria: A population-based cross-sectional study. Int J Infect Dis. 2012;16(2):136-45

21. United Nations, The Millennium Development Goals Report. 2012. [Online]. Available: http://www.un.org/millenniumgoals/pdf/MDG Report 2012.pdf. Accessed 22 Sept 2016.

22. Smith-Greenaway E. Maternal reading skills and child mortality in Nigeria: a reassessment of why education matters. Demography. 2013;50(5):1551-61.

23. Balogun SA, Yusuff HA, Yusuf KQ, Al-Shengiti AM, Balogun MT, Tettey P. Maternal education and child immunization: the mediating roles of maternal literacy and socioeconomic status. Pan Afr Med J. 2017:26:217.

24. Animaw W, Taye W, Merdekios B, Tilahun M, Ayele G. Expanded program of immunization coverage and associated factors among children age 12-23 months in Arba Minch town and Zuria District, Southern Ethiopia, 2013. BMC Public Health. 2014;14(1):464.

25. Bbaale E. Factors influencing childhood immunization in Uganda. J Health Popul Nutr. 2013:31(1):118-29.

26. Branco F, Pereira T, Delfino B, Braña A, Oliart-Guzmán H, Mantovani S, Martins A, de Menezes Oliveira C, Ramalho A, Codeço C, da Silva-Nunes M. Socioeconomic inequalities are still a barrier to full child vaccine coverage in the Brazilian Amazon: a cross-sectional study in Assis Brasil, Acre, Brazil. Int J Equity Health. 2014;13(1):118.

27. Brenner R, Simons-Morton B, Bhaskar B, Das A, Clemens J. Prevalence and predictors of immunization among Inner-City infants: a birth cohort study. Pediatrics. 2001;108(3):661-70.

28. Calhoun L, van Eijk A, Lindblade K, Odhiambo F, Wilson M, Winterbauer E, Slutsker L, Hamel M. Determinants and coverage of vaccination in children in western Kenya from a 2003 cross-sectional survey. Am J Trop Med Hyg. 2013;90(2):234-41.

29. Chhabra P, Nair P, Gupta A, Sandhir M, Kannan A. Immunization in urbanized villages of Delhi. Indian J Pediatr. 2007:74(2):131-4.

30. Elliott C, Farmer K. Immunization status of children under 7 years in the Vikas Nagar area, North India. Child Care Health Dev. 2006;32(4):415-21.

31. Fatiregun A, Okoro A. Maternal determinants of complete child immunization among children aged 12-23 months in a southern district of Nigeria. Vaccine. 2012;30(4):730-6. 
32. Fatiregun A, Adebowale A, Ayoka R, Fagbamigbe A. Assessing full immunisation coverage using lot quality assurance sampling in urban and rural districts of southwest Nigeria. Trans R Soc Trop Med Hyg. 2013;107(11):731-40.

33. Huq MN, Tasnim T. Maternal education and child healthcare in Bangladesh. Matern Child Health J. 2008;12(1):43-51.

34. Jahn A, Floyd S, Mwinuka V, Mwafilaso J, Mwagomba D, Mkisi R, Katsulukuta A, Khunga A, Crampin A, Branson K, McGrath N, Fine P. Ascertainment of childhood vaccination histories in northern Malawi. Tropical Med Int Health. 2008;13(1):129-38.

35. Kidane T, Tekie M. Factors influencing child immunization coverage in a rural District of Ethiopia, 2000. Ethiop J Health Dev. 2003;17(2):105-10.

36. Koumaré A, Traore D, Haidara F, Sissoko F, Traoré I, Dramé S, Sangaré K, Diakité K, Coulibaly B, Togola B, Maïga A. Evaluation of immunization coverage within the expanded program on immunization in Kita circle, Mali: a cross-sectional survey. BMC Int Health Hum Rights. 2009;9(1):S13.

37. Kumar D, Aggarwal A, Gomber S. Immunization status of children admitted to a tertiary-care hospital of north India: reasons for partial immunization or non-immunization. J Health Popul Nutr. 2010;5(2):1215-8.

38. Luman ET, McCauley MM, Shefer A, Chu SY. Maternal characteristics associated with vaccination of young children. Pediatrics. 2003; 111(Supplement 1):1215-8.

39. Mohamud A, Feleke A, Worku W, Kifle M, Sharma H. Immunization coverage of 12-23 months old children and associated factors in Jigjiga District, Somali National Regional State, Ethiopia.BMC Public Health. 2014;14(1):865.

40. Odusanya O, Alufohai E, Meurice F, Ahonkhai V. Determinants of vaccination coverage in rural Nigeria. BMC Public Health. 2008;8(1):381.

41. Okoro J, Ojinnaka N, Ikefuna A, Onyenwe N. Sociodemographic influences on immunization of children with chronic neurological disorders in Enugu, Nigeria. Trials Vaccinology. 2015;4:9-13.

42. Pati S, Feemster K, Mohamad Z, Fiks A, Grundmeier R, Cnaan A. Maternal health literacy and late initiation of immunizations among an Inner-City birth cohort. Matern Child Health J. 2010;15(3):386-94.

43. Phukan R, Barman M, Mahanta J. Factors associated with immunization coverage of children in Assam, India: over the first year of life. J Trop Pediatr. 2008:55(4):249-52.

44. Robert E, Dramaix M, Swennen B. Vaccination coverage for infants: crosssectional studies in two regions of Belgium. Biomed Res Int. 2014;2014:1-7.

45. Rossi R. Do maternal living arrangements influence the vaccination status of children age 12-23 months? A data analysis of demographic health surveys 2010-11 from Zimbabwe. PLoS One. 2015;10(7):e0132357.

46. Schoeps A, Ouédraogo N, Kagoné M, Sié A, Müller O, Becher H. Sociodemographic determinants of timely adherence to BCG, Penta3, measles, and complete vaccination schedule in Burkina Faso. Vaccine. 2013;32(1):96-102.

47. Setse R. HIV-1 infection as a risk factor for incomplete childhood immunization in Zambia. J Trop Pediatr. 2006;52(5):324-8.

48. Singh P, Yadav RJ. Immunization status of children of India. Indian Paediatr. 2000;37(11):1194-9.

49. Singh P, Yadav R. Immunisation status of children in BIMARU states. Indian J Pediatr. 2001;68(6):495-9.

50. Som S, Pal M, Chakrabarty S, Bharati P. Socioeconomic impact on child immunisation in the districts of West Bengal, India. Singap Med J. 2010;51(5):406-12.

51. Streatfield K, Singarimbun M, Diamond I. Maternal education and child immunization. Demography. 1990;27(3):447.

52. Thang NM, Bhushan I, Bloom E, Bonu S. Child immunization in Vietnam: situation and barriers to coverage. J Biosoc Sci. 2006;39(01):41.

53. Torun S, Bakırcı N. Vaccination coverage and reasons for non-vaccination in a district of Istanbul. BMC Public Health. 2006;6(1):125.

54. Waters HR, Dougherty L, Tegang SP, Tran N, Wiysonge CS, Long K, et al. Coverage and costs of childhood immunizations in Cameroon. Bull World Health Organ. 2004;82(9):668-75.

55. Yadav R, Singh P. Immunisation status of children and mothers in the state of Madhya Pradesh. Hindu. 2004;61(77.6):41-5.

\section{Submit your next manuscript to BioMed Central and we will help you at every step:}

- We accept pre-submission inquiries

- Our selector tool helps you to find the most relevant journal

- We provide round the clock customer support

- Convenient online submission

- Thorough peer review

- Inclusion in PubMed and all major indexing services

- Maximum visibility for your research

Submit your manuscript at www.biomedcentral.com/submit
Biomed Central 